\title{
Hypothyroidism in patients with hepatocellular carcinoma receiving cabozantinib: an unassessed issue
}

\author{
Vincenzo Di Nunno ${ }^{\ddagger 1}$, Giorgio Frega ${ }^{\ddagger}$ 2, Lidia Gatto ${ }^{\ddagger 2}$, Giovanni Brandi ${ }^{\ddagger}, 2$ \& Francesco \\ Massari $*, \neq \ddagger, 1$ \\ 'Division of Oncology, Sant'Orsola-Malpighi Hospital, Bologna, Italy \\ ${ }^{2}$ Department of Experimental, Diagnostic \& Specialty Medicine, Oncology Unit, Sant'Orsola-Malpighi Hospital, University of \\ Bologna, Bologna, Italy \\ *Author for correspondence: fmassari79@gmail.com \\ $¥$ Co-first authors \\ $\$$ Co-last authors
}
"Gthyroid function should be periodically assessed in patients with advanced/metastatic HCC receiving cabozantinib as a substitution treatment could quickly correct this adverse event preventing more severe symptoms related to this condition."

First draft submitted: 1 November 2018; Accepted for publication: 9 November 2018; Published online: 16 January 2019

In the last decades, several innovative systemic therapies except for sorafenib (e.g., sunitinib, brivanib, erlotinib and so on) failed to improve overall survival in hepatocellular carcinoma (HCC) patients not eligible for surgery or locoregional procedures [1].

More recently, other targeted therapies (tyrosine kinase inhibitor or monoclonal antibodies) showed some activity in randomized Phase III trials both in first-line (lenvatinib) and in second-line (regorafenib, cabozantinib and ramucirumab) treatment [2-5].

Lenvatinib is a tyrosine kinase inhibitor of the VEGFR 1-3, FGFRs 1-4, platelet-derived growth factor receptor, rearranged during transfection and stem cell growth factor receptor (KIT). In a Phase III noninferiority trial lenvatinib showed nonsurvival inferiority (13.6 vs 12.3 months; hazard ratio [HR]: 0.92, 95\% CI: 0.70-1.06) compared with sorafenib in the first-line setting [3]. In the REACH trial, the recombinant monoclonal antibody ramucirumab targeting VEGFR2 failed to improve overall survival over placebo in previously treated patients with metastatic HCC (HR: 0.87, 95\% CI: 0.72-1.05) [4].

More positive results emerged from the RESORCE trial in which 573 patients with HCC who tolerated sorafenib and progressed to sorafenib maintaining a Child Pugh A liver function were randomized to receive regorafenib or placebo. Patients who received the multityrosine kinase inhibitor regorafenib achieve a longer survival (10.6 vs 7.8 months; HR: 0.63, 95\% CI: 0.5-0.79). However, toxicity was significant in the regorafenib arm with hypertension, hand-foot skin reaction, fatigue and diarrhea as the most frequent grade 3-4 treatment-related events. Of note seven deaths $(2 \%)$ were considered as related to regorafenib [5].

Increasing evidence shows that HCC is a tumor with high immunological potential due to the expression of tumor-associated antigens and specific neoantigens arising from specific mutations. However, due to the interaction of several immune-inhibitory molecules resulting from Treg and stroma cells interaction, immune response against the tumor is often suppressed [6]. Due to these issues, immune checkpoint inhibitors are a promising systemic approach in HCC and so, unsurprisingly, several ongoing trials are testing these agents in different settings of the disease.

Nivolumab (an anti PD-1 monoclonal antibody) led to encouraging results in terms of response rates and survival, in a Phase I-II study (CheckMate 040 trial) including patients pretreated with sorafenib [7]. Indeed, in this Phase I/II trial the objective response rate was $20 \%$ in the nivolumab $3 \mathrm{mg} / \mathrm{kg}$ dose expansion phase while a $15 \%$ overall response rate (ORR) has been observed in the dose-escalation phase. Furthermore, nivolumab showed a safety profile with no treatment-related deaths. 
Still in the second-line setting, promising results were obtained with pembrolizumab in a nonrandomized, multicenter, open-label, Phase II trial (KEYNOTE-224), and with durvalumab in a Phase I-II trial. They both demonstrated a hopeful antitumor activity $(17$ and $10 \%$ in ORR, respectively) $[8,9]$. Further trials with immunotherapies in the first-line setting are currently ongoing.

In this scenario we welcomed the results of the CELESTIAL Phase III trial in which cabozantinib was demonstrated to improve survival, progression-free survival and response rate of patients with advanced HCC progressed to systemic treatment [2]. Cabozantinib is an oral multityrosine kinase inhibitor that targets different pathways including the VEGFR, mesenchymal-epithelial transition (MET) and Anexelekto. These pathways interact with each other to promote angiogenesis and tumor progression [10,11]. Of interest, MET seems to be related to tumor dedifferentiation and clinical aggressiveness. Moreover, further evidences seem to correlate MET hyperexpression to bone invasion in renal cell carcinoma (RCC) [10]. MET expression increases after exposure to antiangiogenic factors such as sunitinib or sorafenib, suggesting that probably this receptor is involved in mechanisms related to VEGF/VEGFR inhibitor resistance [10-12].

To date, cabozantinib is adopted in advanced medullary thyroid cancer and in metastatic renal cell carcinoma. In the already cited CELESTIAL trial, 707 patients with previously treated advanced HCC were randomized (2:1) to receive cabozantinib at the standard dose of $60 \mathrm{mg}$ daily or placebo. Of note, all patients progressed to sorafenib and may have received up to two previous systemic regimens. Patients who received cabozantinib demonstrated a significantly longer overall survival (10.2 vs 8.0 months; HR: $0.76,95 \%$ CI: $0.63-0.92)$, progression-free survival (5.2 vs 1.9 months; HR: $0.44,95 \%$ CI: $0.36-0.52)$ and better ORR ( 4 vs $<1 \%$ ). About $68 \%$ of patients in cabozantinib group experienced grade 3 or 4 adverse events with palmar-plantar erythrodysesthesia (17\%), hypertension (16\%) increased aspartate aminotransferase level (12\%), fatigue (10\%) and diarrhea (10\%) as the most common high-grade toxicity events.

Due to these remarkable results, there are few doubts that cabozantinib will change our clinical practice. Considering other trials exploring this molecule, it could be interesting to observe that the toxicity profile is largely conserved across these diseases with the exception of hypothyroidism.

Hypothyroidism, resulting from cabozantinib administration, is not a negligible toxicity, but was not observed in the CELESTIAL trial [2,13-16].

The recognition of this adverse event is of particular interest as some of the hypothyroidism symptoms could be easily interpreted as symptoms related to disease. Dry skin and hair, cold sensitivity, weight gain, fatigue, muscle cramps, voice changes, constipation, carpal tunnel syndrome, sleep apnea, depression, irritability, pituitary hyperplasia, hyponatremia and bradycardia are common manifestations of hypothyroidism. Diagnosis of this condition required laboratory dosage of free thyroxine (fT4), free triiodothyronine (fT3), thyroid-stimulating hormone as well as ultrasound imaging of thyroid gland.

In other trials exploring cabozantinib, G1-2 hypothyroidism has been observed as a common toxicity in RCC and prostate cancer, while this toxicity has not been reported in trials carried out on medullary thyroid cancer and HCC patients. Of note, G3-4 thyroid toxicities have rarely been observed. This is mainly due also to an early recognition across clinical trials exploring thyroid function, which allowed the administration of an appropriate supplementation of thyroid hormone.

Regarding RCC, hypothyroidism has been observed in the METEOR trial in which patients often received sunitinib as a previous treatment. As it is well known, sunitinib is a tyrosine kinase inhibitor whose administration often results in hypothyroidism and so this previously exposed patient could have influenced the toxicity observed. However, cabozantinib-related hypothyroidism has also been observed in the first-line setting in the CABOSUN trial in sunitinib-naive patients.

Furthermore, a study confirmed thyroid toxicity of cabozantinib also in bladder tumors and sarcoma [17,18].

Although thyroid dysfunction is commonly observed among trials adopting tyrosine kinase inhibitors, the mechanisms by which this occurs remain poorly understood. It is possible that thyroid damage occurs due to different mechanisms including: reduced synthesis of thyroid hormone, inhibition of iodide uptake, transmembrane transport protein deficit, impairment of deiodinase enzymes, or autoimmune/ischemic damage. Furthermore, during tyrosine kinase administration an increased dose of levothyroxine replacement is sometimes required in patients who underwent thyroidectomy, suggesting that these agents may also interact with levothyroxine absorption [17]. Of note, hypothyroidism can be a transient condition occurring during tyrosine kinase inhibitor administration as well as a permanent condition resulting from thyroid gland damage. 
Although hypothyroidism has not emerged as common adverse event in CELESTIAL trial it is probable that longer follow-up will reveal this common toxicity also in HCC patients. As patients enrolled in this trial were previously treated with sorafenib it is possible that thyroid damage resulted from this treatment. However, administration of cabozantinib could also lead to hypothyroidism in patients with normal thyroid function, and even if this toxicity is generally subclinical its recognition is important as patients with hypothyroidism could get a worse clinical outcome and it could negatively influence their quality of life.

So considering these issues thyroid function should be periodically assessed in patients with advanced/metastatic HCC receiving cabozantinib as a substitution treatment could quickly correct this adverse event preventing more severe symptoms related to this condition.

\section{Financial \& competing interests disclosure}

The authors have no relevant affiliations or financial involvement with any organization or entity with a financial interest in or financial conflict with the subject matter or materials discussed in the manuscript. This includes employment, consultancies, honoraria, stock ownership or options, expert testimony, grants or patents received or pending, or royalties.

No writing assistance was utilized in the production of this manuscript.

\section{Reference}

1 Llovet JM, Montal R, Sia D, Finn RS. Molecular therapies and precision medicine for hepatocellular carcinoma. Nat. Rev. Clin. Oncol. 15(10), 599-616 (2018).

2 Abou-Alfa GK, Meyer T, Cheng AL et al. Cabozantinib in patients with advanced and progressing hepatocellular carcinoma. N. Engl. J. Med. 379(1), 54-63 (2018).

3 Kudo M, Finn RS, Qin S et al. Lenvatinib versus sorafenib in first-line treatment of patients with unresectable hepatocellular carcinoma: a randomised Phase III non-inferiority trial. Lancet 391(10126), 1163-1173 (2018).

4 Zhu AX, Park JO, Ryoo BY et al. Ramucirumab versus placebo as second-line treatment in patients with advanced hepatocellular carcinoma following first-line therapy with sorafenib (REACH): a randomised, double-blind, multicentre, Phase III trial. Lancet Oncol. 16(7), 859-870 (2015).

5 Bruix J, Qin S, Merle P et al. Regorafenib for patients with hepatocellular carcinoma who progressed on sorafenib treatment (RESORCE): a randomised, double-blind, placebo-controlled, Phase III trial. Lancet 389(10064), 56-66 (2017).

6 Prieto J, Melero I, Sangro B. Immunological landscape and immunotherapy of hepatocellular carcinoma. Nat. Rev. Gastroenterol. Hepatol. 12(12), 681-700 (2015).

7 El-Khoueiry AB, Sangro B, Yau T et al. Nivolumab in patients with advanced hepatocellular carcinoma (CheckMate 040): an open-label, non-comparative, Phase I/II dose escalation and expansion trial. Lancet 389(10088), 2492-2502 (2017).

8 Zhu AX, Finn RS, Edeline J et al. Pembrolizumab in patients with advanced hepatocellular carcinoma previously treated with sorafenib (KEYNOTE-224): a non-randomised, open-label Phase II trial. Lancet Oncol. 19(7), 940-952 (2018).

9 Zev AW, Neil HS, Dirk J et al. Safety and clinical activity of durvalumab monotherapy in patients with hepatocellular carcinoma (HCC). J. Clin. Oncol. 35(15 Suppl.), 4071-4071 (2017).

10 Di Nunno V, Cimadamore A, Santoni M et al. Biological issues with cabozantinib in bone metastatic renal cell carcinoma and castration-resistant prostate cancer. Future Oncol. 14(25), 2559-2564 (2018).

11 Di Nunno V, Cubelli M, Massari F. The role of the MET/AXL pathway as a new target for multikinase inhibitors in renal cell carcinoma. Expert Rev. Precision Med. Drug Dev. 2(3), 169-175 (2017).

12 Rimassa L, Abbadessa G, Personeni N et al. Tumor and circulating biomarkers in patients with second-line hepato-cellular carcinoma from the randomized Phase II study with tivantinib. Oncotarget 7, 72622-72633 (2016).

13 Choueiri TK, Escudier B, Powles T et al. Cabozantinib versus everolimus in advanced renal-cell carcinoma. N. Engl. J. Med. 373(19), 1814-1823 (2015).

14 Choueiri TK, Halabi S, Sanford BL et al. Cabozantinib versus sunitinib as initial targeted therapy for patients with metastatic renal cell carcinoma of poor or intermediate risk: the alliance A031203 CABOSUN Trial. J. Clin. Oncol. 35(6), 591-597 (2017).

15 Elisei R, Schlumberger MJ, Müller SP et al. Cabozantinib in progressive medullary thyroid cancer. J. Clin. Oncol. 31(29), 3639-3646 (2013).

16 Smith M, De Bono J, Sternberg C et al. Phase III study of cabozantinib in previously treated metastatic castration-resistant prostate cancer: COMET-1. J. Clin. Oncol. 34(25), 3005-3013 (2016).

17 Yavuz S, Apolo AB, Kummar S et al. Cabozantinib-induced thyroid dysfunction: a review of two ongoing trials for metastatic bladder cancer and sarcoma. Thyroid 24(8), 1223-1231 (2014).

18 Liwanpo L, Hershman JM. Conditions and drugs interfering with thyroxine absorption. Best Pract. Res. Clin. Endocrinol. Metab. 23, 781-792 (2009). 